\title{
XMMSL1 J074008.2-853927: a tidal disruption event with thermal and non-thermal components ${ }^{\star}$
}

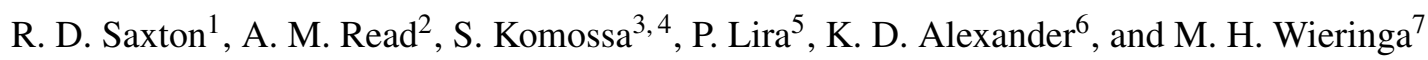 \\ 1 XMM SOC, ESAC, Apartado 78, 28691 Villanueva de la Cañada, Madrid, Spain \\ e-mail: richard.saxton@sciops.esa.int \\ 2 Dept. of Physics and Astronomy, University of Leicester, Leicester LE1 7RH, UK \\ 3 QianNan Normal University for Nationalities, Longshan Street, Duyun City of Guizhou Province, PR China \\ ${ }_{5}^{4}$ Max Planck Institut für Radioastronomie, Auf dem Huegel 69, 53121 Bonn, Germany \\ 5 Universidad de Chile, Observatorio Astronomico Nacional Cerro Calan, Santiago, Chile \\ 6 Harvard-Smithsonian Center for Astrophysics, 60 Garden St., Cambridge, MA 02138, USA \\ 7 Australia Telescope National Facility, CSIRO Astronomy and Space Science, PO Box 76, Epping, NSW 1710, Australia
}

Received 27 May 2016 / Accepted 29 September 2016

\begin{abstract}
Aims. We study X-ray bright tidal disruption events (TDE), close to the peak of their emission, with the intention of understanding the evolution of their light curves and spectra.

Methods. Candidate TDE are identified by searching for soft X-ray flares from non-active galaxies in recent XMM-Newton slew data. Results. In April 2014, X-ray emission was detected from the galaxy XMMSL1 J074008.2-853927 (a.k.a. 2MASX 074007858539307), a factor 20 times higher than an upper limit from 20 years earlier. Both the X-ray and UV flux subsequently fell, by factors of 70 and 12 respectively. The bolometric luminosity peaked at $L_{\mathrm{bol}} \sim 2 \times 10^{44} \mathrm{ergs} \mathrm{s}^{-1}$ with a spectrum that may be modelled with thermal emission in the UV band, a power-law with $\Gamma \sim 2$ dominating in the X-ray band above $2 \mathrm{keV}$ and a soft X-ray excess with an effective temperature of $\sim 86 \mathrm{eV}$. Rapid variability locates the X-ray emission to within $<73 R_{\mathrm{g}}$ of the nuclear black hole. Radio emission of flux density $\sim 1 \mathrm{mJy}$, peaking at $1.5 \mathrm{GHz}$ was detected 21 months after discovery. Optical spectra indicate that the galaxy, at a distance of $73 \mathrm{Mpc}(z=0.0173)$, underwent a starburst $2 \mathrm{Gyr}$ ago and is now quiescent. We consider a tidal disruption event to be the most likely cause of the flare. If this proves to be correct then this is a very clean example of a disruption exhibiting both thermal and non-thermal radiation.
\end{abstract}

Key words. X-rays: galaxies - galaxies: individual: XMMSL1 J074008.2-853927

\section{Introduction}

When a star approaches very close to a supermassive black hole $(\mathrm{SMBH})$ the gravitational shear can overcome the selfgravitation of the star causing it to be torn apart (Hills 1975) in what is known as a tidal disruption event (TDE). About half of the stellar debris remains gravitationally bound to the hole and is accreted on return, causing a flare of thermal radiation which peaks in the EUV band (Rees 1988). This radiation signature has been observed in the soft X-ray (Komossa et al. 2004; Bade et al. 1996; Komossa \& Greiner 1999b; Esquej et al. 2007; Saxton et al. 2012; Maksym et al. 2010; Lin et al. 2015) and UV (Gezari et al. 2006, 2008, 2009) bands. Optical flares have also been detected (van Velzen et al. 2011; Cenko et al. 2012; Gezari et al. 2012; Arcavi et al. 2014) from candidate TDE, with an apparent temperature of a few $\times 10^{4} \mathrm{~K}$, cooler than that expected from an accretion disc (e.g. Bonning et al. 2007). Current models for the optical emission centre on a thick screen of debris, which reprocesses the inner thermal radiation and reemits it in the optical band (Strubbe \& Quataert 2009, 2011; Guillochon et al. 2014). Strubbe \& Murray (2015) suggest that the initial accretion phase will be highly super-Eddington, resulting in a massive radiation-driven outflow of material that will absorb the radiation from the central engine and convert it into

\footnotetext{
* Data for this object are available within the Open TDE Catalog at https://tde. space/tde/XMMSL1J0740-85
}

optical photons. At a later stage, the screen density will drop and the inner thermal radiation will become visible. A further signature of reprocessing is given by broad, low-ionisation optical lines which indicate a large solid angle of absorbing material orbiting the nucleus. Komossa et al. (2008) discovered superstrong broad hydrogen, helium and highly ionised narrow Iron lines which faded over time in SDSS J095209.56+214313.3. Bright fading optical broad lines were also seen in other archival SDSS data (Wang et al. 2011) and in ASSASN-14li (Holoien et al. 2016a). Broad nitrogen and helium lines were also detected in the UV band from ASSASN-14li (Cenko et al. 2016). The rate at which galaxies produce a soft X-ray TDE has been estimated from ROSAT observations as $9 \times 10^{-6}$ events/galaxy/year (Donley et al. 2002); from XMM-Newton slew observations as $2 \times 10^{-4}$ events/galaxy/year (Esquej et al. 2008) and from galaxy clusters $1.2 \times 10^{-4}$ events/galaxy/year (Maksym et al. 2010). These numbers are comparable to the rate of TDE, detected from their peak optical emission of $2 \times 10^{-5}$ events/galaxy/year (van Velzen \& Farrar 2014). Even though these estimates are based on small numbers of events, they are difficult to reconcile with a model where the majority of $\mathrm{X}$-ray events are invisible due to a thick absorber. Furthermore, strong two-temperature soft X-ray and optical emission has been observed simultaneously from the well-monitored TDE, ASSASN-14li, implying that our view to the centre is rather unobscured, apart from a highly-ionised, slowly-expanding, screen 
of material with a column of $\sim 10^{23} \mathrm{~cm}^{-2}$ (Miller et al. 2015; Cenko et al. 2016). Finally, the exceptional X-ray softness of the first identified TDEs strongly argues against large amounts of obscuring material (e.g. Komossa \& Bade 1999a).

Radio emission has been associated with TDE in several events. Swift J164449.3+573451 exhibited strong, variable radio emission compatible with a forward shock from material swept-up by an on-axis relativistic jet (Zauderer et al. 2011). This was also seen in the more distant object Swift 2058+0516 (Cenko et al. 2012). Both of these were discovered from their super-Eddington, hard ( $>2 \mathrm{keV}$ ) X-ray emission by Swift, as was a further jetted event, Swift 1112-8238 (Brown et al. 2015). Hard X-ray emission dominated the spectrum of NGC 5485 (Nikolajuk \& Walter 2013), also accompanied by radio emission (Irwin et al. 2015) which led to the suggestion that this was another relativistic event, this time viewed off-axis (Lei et al. 2016). Transient radio emission was seen in ASSASN14li (Alexander et al. 2016; van Velzen et al. 2016) but at a level which may be consistent with a non-relativistic outflow rather than a jet. It is an open, and interesting, question as to why these events should show a hard X-ray component, compatible with power-law emission, while many TDE show only thermal X-ray emission (e.g. Komossa 2002; Esquej et al. 2007; Saxton et al. 2012; Maksym et al. 2010; Lin et al. 2015; Holoien et al. 2016a). In 2MASS 0619-65 (Saxton et al. 2014) hard X-ray emission was not accompanied by a radio flare and it is not yet decided whether the high-energy photons, which are seen in TDE come from a jet or from the hot, comptonising, electron plasma which is believed to be responsible for the same component in AGN (Sunyaev \& Titarchuk 1980; Haardt \& Maraschi 1991). Even in Swift J164449.3+573451, evidence for X-ray reprocessing within a few gravitational radii $\left(R_{\mathrm{g}}\right)$ of the black hole has called into question the hitherto accepted view that the huge $\mathrm{X}$-ray luminosity $\left(L_{\mathrm{X}} \sim 10^{48} \mathrm{ergs} \mathrm{s}^{-1}\right)$ was produced by a jet (Kara et al. 2016).

In April 2014, the XMM-Newton slew survey (Saxton et al. 2008) detected a flare from the nucleus of the quiescent galaxy XMMSL1 J074008.2-853927 which further monitoring showed to be both thermal and non-thermal, having simultaneous flares in the UV, soft X-ray and hard X-ray bands. In Sect. 2 we discuss the discovery of this flare and the source identification; in Sects. 3-5 we present X-ray, UV, optical and radio follow-up observations; in Sect. 6 we perform a temporal and spectral analysis of the source and in Sect. 7 we discuss the flare characteristics within the TDE model. The paper is summarised in Sect. 8.

A $\Lambda$ CDM cosmology with $\left(\Omega_{\mathrm{M}}, \Omega_{\Lambda}\right)=(0.27,0.73)$ and $H_{0}=$ $70 \mathrm{~km}^{-1} \mathrm{~s}^{-1} \mathrm{Mpc}^{-1}$ has been assumed throughout.

\section{X-ray flare identification}

During the slew 9262100003, performed on April 1st 2014, XMM-Newton (Jansen et al. 2001) detected a source, XMMSL1 J074008.2-853927 (hereafter XMMSL1 J0740-85), with an EPIC-pn, medium filter, 0.2-2 keV count rate of $4.2 \pm$ 0.6 count $\mathrm{s}^{-1}$. A crude analysis may be performed on the 45 photons in the slew spectrum to investigate the gross spectral properties of the detection. Detector matrices were calculated, taking into account the transit of the source across the detector, using a technique outlined in Read et al. (2008). A simple absorbed power-law fit gives a slope $\Gamma=2.9 \pm 0.5$ assuming no intrinsic absorption above the Galactic value of $1.5 \times 10^{21} \mathrm{~cm}^{-2}$ (Willingale et al. 2013). This corresponds to an absorbed flux of $F_{0.2-2} \sim 4.9 \pm 0.7 \times 10^{-12} \mathrm{ergs} \mathrm{s}^{-1} \mathrm{~cm}^{-2}$ using the above model.
The count rate in the $2-10 \mathrm{keV}$ band is $0.8 \pm 0.3$ count $\mathrm{s}^{-1}$. Using the best spectral model from follow-up observations this gives a flux, $F_{2-10} \sim 4.8 \pm 1.8 \times 10^{-12} \mathrm{ergs} \mathrm{s}^{-1} \mathrm{~cm}^{-2}$. We calculate a 2-sigma upper limit from the ROSAT All-Sky Survey (RASS) at this position of 0.021 count s$^{-1}$ (see Esquej et al. 2007, for a description of the upper limit calculation); a factor 20 lower $0.2-2 \mathrm{keV}$ flux using the same spectral model.

The source position lies $3.8^{\prime \prime}$ from the galaxy 2MASX 07400785-8539307 $(z=0.0173$; Huchra et al. 2012) which with $J=11.92 \pm 0.03, H=11.15 \pm 0.04$, $K=10.96 \pm 0.05, R=10.7$ is the only bright optical and infrared source within the $8^{\prime \prime}$ error circle (Saxton et al. 2008).

\section{X-ray and UV observations}

An X-ray monitoring program was initiated with Swift (Gehrels et al. 2004) to follow the evolution of the source flux and spectrum. Snapshot $3 \mathrm{ks}$ observations were made, initially once a week and then less frequently, with the Swift-XRT (Burrows et al. 2005) in photon counting mode and the UV optical telescope (UVOT; Roming et al. 2005). The Swift-XRT observations have been analysed following the procedure outlined in Evans et al (2009) and the UVOT data have been reduced as described in Poole et al. (2008). An accurate position for the source in the Swift-XRT field can be determined by matching the UVOT field of view with the USNO-B1 catalogue and registering the XRT field accordingly (Goad et al. 2007). The resulting source position, $\alpha_{\mathrm{J} 2000}=07: 40: 08.28, \delta_{\mathrm{J} 2000}=-85: 39: 32.4$ $\left( \pm 2.0^{\prime \prime} ; 90 \%\right.$ confidence $)$ is coincident with the galactic nucleus (see Fig. 1). A more precise position is available from the UVOT images. The source position from the first Swift-UVOT observation, which used the uvw2 filter, is $\alpha_{\mathrm{J} 2000}=07: 40: 08.43$, $\delta_{\mathrm{J} 2000}=-85: 39: 31.4$ with an error dominated by a systematic offset of $\pm 0.42^{\prime \prime}$ (90\% confidence; Breeveld et al. 2010). This is consistent, within the error circle, with the galactic centre given by the 2MASS point source catalogue $\left(\alpha_{\mathrm{J} 2000}=07: 40: 08.09\right.$, $\left.\delta_{\mathrm{J} 2000}=-85: 39: 31.3\right)$. We can conclude that the flare was consistent with being from the nucleus of 2MASX 07400785-8539307 to within the accuracy of the UVOT pointing (150 pc at the galaxy redshift).

In parallel, XMM-Newton pointed observations were triggered on 2014-04-30 (due to scheduling issues this was split into three parts; obsid $=0740340301,0740340401,0740340501$ ) and 2015-01-12 (obsid =0740340601). A summary of observations and exposure times is given in Table 1 . In each observation, the EPIC-pn and MOS-1 cameras were operated in full frame mode with the thin 1 filter in place, while the MOS-2 camera was used in small window mode with the medium filter. The source was too faint for statistically significant data to be collected from the reflection grating spectrometers.

The XMM data were analysed with the XMM-Newton Science Analysis System (SAS v14.0.0; Gabriel et al. 2003). Light curves were extracted from the observations and searched for periods of high background flaring. These periods were excluded from the datasets leaving for the first observation a total of $26.6 \mathrm{ks}$ of useful data and for the second observation $16.1 \mathrm{ks}$.

\section{Optical observations}

XMMSL1 J0740-85 was observed by the CTIO $1.5 \mathrm{~m}$ telescope, on 2002-01-17, as part of the 2MASS redshift survey (Huchra et al. 2012). The optical spectrum showed a non-active galaxy (Fig. 2) at a redshift of $z=0.0173$. We calibrated the 
Table 1. X-ray observation log of XMMSL1 J0740-85.

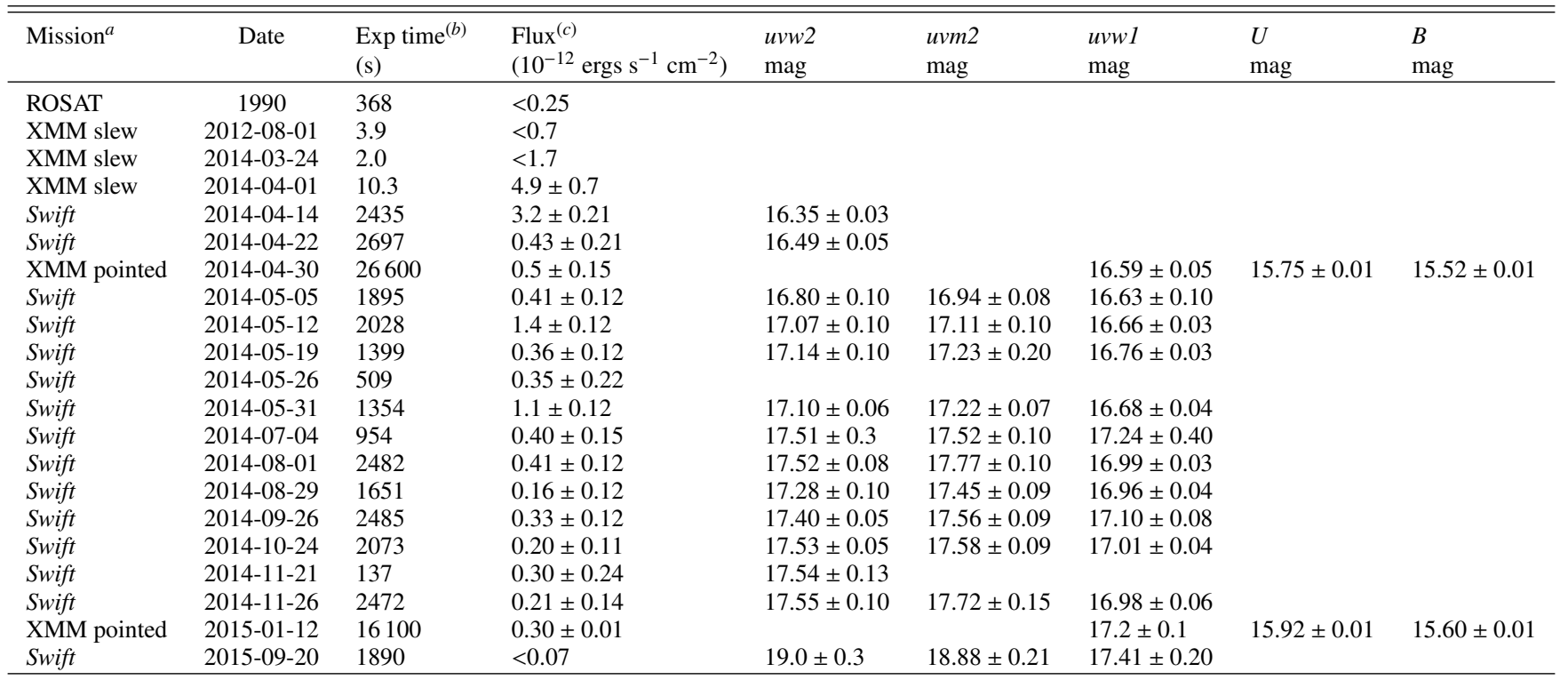

Notes. ${ }^{(a)}$ XMM-Newton, EPIC-pn camera: slew observations performed in full frame mode with the medium filter; pointed observations performed in full frame mode with the thinl filter. Swift-XRT observations performed in $p c$ mode. ROSAT sky survey data taken between 1990-08-12 and 1991-02-18. ${ }^{(b)}$ Exposure time in seconds. For the two XMM pointed observations, this is the remaining exposure after removing times of high background flares. ${ }^{(c)}$ Absorbed flux in the $0.2-2 \mathrm{keV}$ band, calculated by fitting the spectrum of each observation with a two-component model of a power-law plus black-body absorbed by a Galactic column of $1.5 \times 10^{21} \mathrm{~cm}^{-2}$. Upper limits have been calculated using a power-law model of slope 2.9 absorbed by the Galactic column.

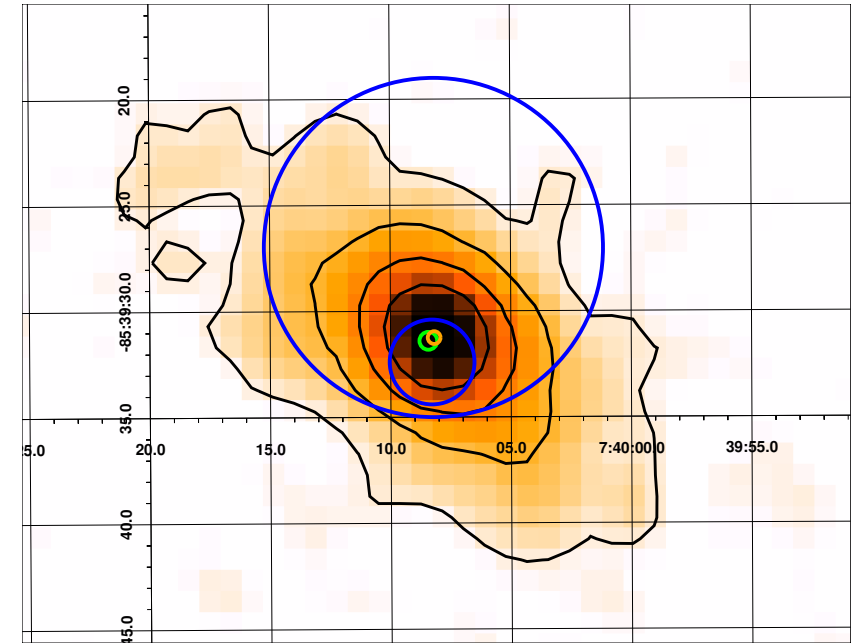

Fig. 1. A 2MASS image of the galaxy, shown with the XMM-Newton slew (blue, $1-\sigma, 8^{\prime \prime}$ radius), UVOT-enhanced Swift-XRT (blue, 90\% conf., 2.0" radius; see text), Swift-UVOT (green, $90 \%$ conf., $0.42^{\prime \prime}$ radius) and ATCA, radio (orange, $1-\sigma, 0.3^{\prime \prime}$ radius) error circles, centred on the detections.

raw spectrum using the spectrophotometric standards, LTT 377 and LTT 3864. Observations at CTIO were obtained at 1.8 airmasses using a fixed E-W slit orientation (Macri, priv. comm.), which might introduce slit losses at the blue end due to atmospheric differential refraction. However, for the given airmass, the refraction at $4000 \AA$ with respect to the position of the image at $5000 \AA$ corresponds to $\sim 0.95$ arcsec (Filippenko 1982). This is less than half the used slit width, and therefore differential refraction should not greatly impact the observation.

We reobserved the source with the WFCCD imagerspectrograph mounted on the du Pont 100-inch telescope located at Las Campanas Observatory (LCO) in Chile on the 4th of May 2014. The spectrograph blue grism was used resulting in a spectral resolution of $\sim 8 \AA$. Integration time was $600 \mathrm{~s}$. The data were reduced using IRAF packages in the standard way. Wavelength calibration was achieved using HeArNe lamps, while flux calibration was achieved using the observation of the LTT4364 spectrophotometric standard star. Even though the spectral range of the observations extended to $9000 \AA$, the analysis was conducted only in the 3895-7150 $\AA$ spectral window due to telluric absorption and heavy fringing in the red end.

The red end of the spectrum obtained from LCO is 1.55 times brighter than that obtained from CTIO, which may be explained by the different slit widths of $4.8^{\prime \prime}$ and $2.6^{\prime \prime}$ used respectively in each observation. In Fig. 2 we compare the two calibrated spectra, after scaling the CTIO spectrum to match the LCO observations, and find that the main difference is an increase in blue flux in the later observation below $\sim 4800 \AA$. The difference spectrum is featureless apart from a spurious absorption feature at $\sim 4300 \AA$ in the CTIO spectrum which appears as a spike in the difference spectrum.

The stellar population of XMMSL1 J0740-85 was analysed using the STARLIGHT spectral population synthesis code (Cid Fernandes et al. 2005, 2011) on the CTIO spectrum. The code uses the Bruzual \& Charlot (2003) single stellar population models and requires the data to be corrected for foreground Galactic extinction and taken to the rest frame. We used the Cardelli et al. (1989) extinction curve to correct for foreground reddening and adopted $A_{V}=0.437$ and shifted the spectrum to the rest wavelength using $z=0.017292$. Figure 3 presents the results from the spectral population synthesis analysis. The bottom panel shows the observed and total synthetic spectra and the fit residuals. The histograms present the percentage that each of the single stellar populations provides to the current total mass and $B$-band flux of the galaxy. The fit shows that the stellar 


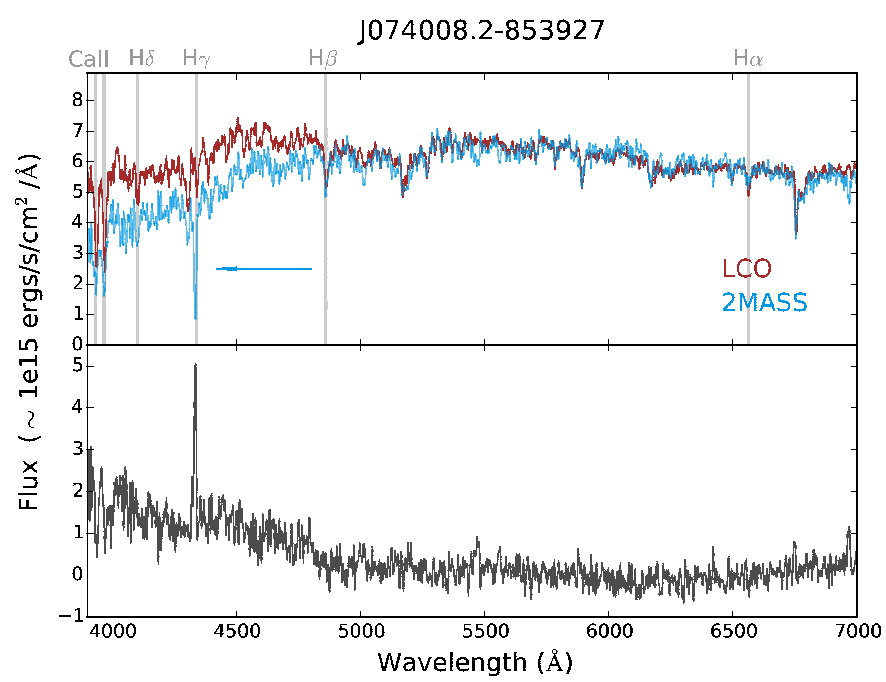

Fig. 2. Optical spectra of XMMSL1 J0740-85 taken on 2002-01-17 with the CTIO $1.5 \mathrm{~m}$ telescope for the 2MASS redshift survey (blue) and on 2014-05-05 with the Las Campanas, du Pont 100-inch telescope (red). The CTIO spectrum has been scaled by a factor 1.55 to match the Las Campanas spectrum at the red end. The lower panel shows the difference spectrum, indicating a featureless excess in blue flux in the 2014 observation. The apparent spike in the difference spectrum at $\sim 4300 \AA$ is caused by a spurious absorption feature in the CTIO spectrum.
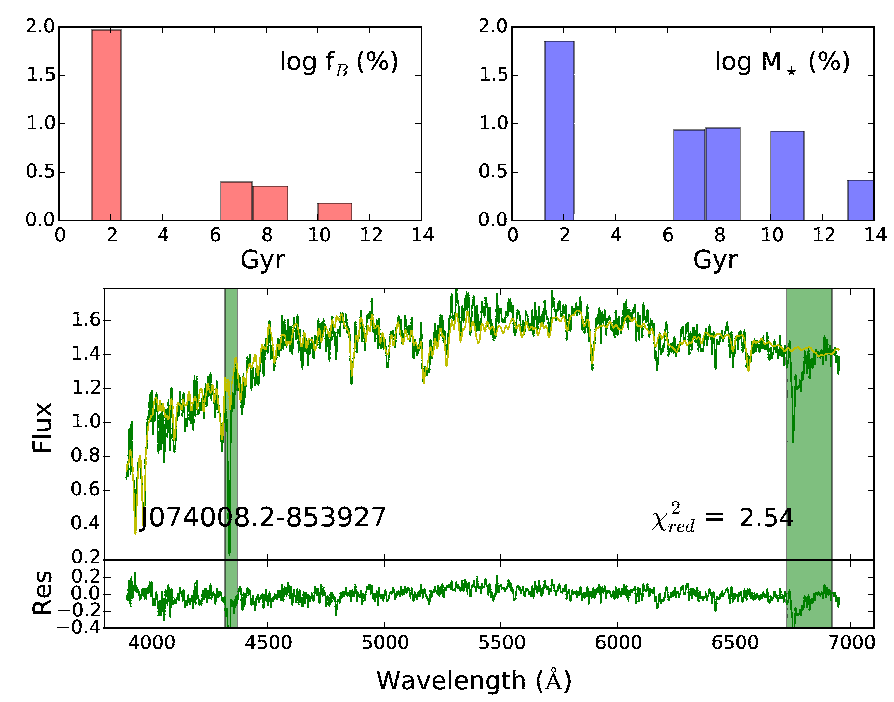

Fig. 3. A stellar population fit of XMMSL1 J0740-85 to the 200201-17 CTIO optical spectrum, with the fitted age of stellar populations providing the blue flux (upper left) and the total mass (upper right). Residuals to the stellar fit are shown in the bottom panel. See text for details of the fitting procedure.

emission is dominated by a relatively young stellar population of age 2 Gyr but that a significant amount of mass could be found in a much older population. The integrated absolute $J$ magnitude of J0740 is -22.5 , which is about 1 mag below the knee of the J-band local galaxy luminosity (Cole et al. 2001). It is likely that, in such a massive galaxy, most of the stellar mass was formed at rather high redshifts, and this component would most likely be hidden under the light of the 2 Gyr old component.

The residuals clearly show that no emission lines are present in XMMSL1 J0740-85 with a strict upper limit to the OIII emission line flux of $4 \times 10^{-15} \mathrm{ergs} \mathrm{s}^{-1}$.

\section{Radio observation}

We observed the position of XMMSL1 J0740-85 with the Australia Telescope Compact Array (ATCA) on 2015-11-14 UT in the $4 \mathrm{~cm}$ band and 2015-12-01 UT in the $16 \mathrm{~cm}$ and $15 \mathrm{~mm}$ bands. We detected a source at $\alpha_{\mathrm{J} 2000}=07: 40: 08.19$, $\delta_{\mathrm{J} 2000}=-85: 39: 31.25\left( \pm 0.3^{\prime \prime}\right)$, which is consistent with the XMM-Newton and Swift positions. The source is detected at all frequencies, with a peak flux density of $1.19 \pm 0.06 \mathrm{mJy}$ at $1.53 \mathrm{GHz}$. At a distance of $73 \mathrm{Mpc}$, this corresponds to a luminosity $L_{v}=(7.7 \pm 0.4) \times 10^{27} \mathrm{erg} \mathrm{s}^{-1} \mathrm{~Hz}^{-1}$. This is slightly lower than the radio luminosity of the nearby thermal TDE ASSASN-14li (likely produced by a non-relativistic outflow; Alexander et al. 2016; van Velzen et al. 2016), an order of magnitude below the radio luminosity of the candidate TDE IGR J12580+0134 (possibly due to an off-axis jet; Irwin et al. 2015; Lei et al. 2016), and several orders of magnitude below the radio luminosities of relativistic TDEs like Swift J164449.3+573451 (ascribed to an on-axis relativistic jet; Zauderer et al. 2011). Additional radio observations of XMMSL1 J0740-85 are planned and the time-evolution of the emission will be discussed in a future paper (Alexander et al., in prep.).

\section{X-ray and UV variability}

In Fig. 4 we show the historical light curve of XMMSL1 J0740-85. All bands show gradually decreasing flux with the largest decrement being in the highest-energy bands. In principle, the date of peak emission can be constrained to be between an XMM-Newton slew survey non-detection of 2014-03-24 and the detection on 2014-04-01 with a soft X-ray flux three times higher than this upper limit. Nevertheless, we see from this and other TDE (Saxton et al. 2012; Levan et al. 2011) that the light curves can be very variable in the early phase and so the actual peak may have occurred up to a few weeks earlier then the 2014-04-01 observed peak. The soft and hard X-ray light curves may be adequately hand-fit by the decay model predicted by the classical model of TDE (Rees 1988; Phinney 1989) of $L \propto\left(t-t_{0}\right)^{5 / 3}$ with a peak time, $t_{0}=35$ days before the first detection (2014-02-25; see Fig. 5).

In Fig. 6 we show the short-term light curve from the two XMM-Newton pointed observations. The X-ray flux, from the first XMM observation, shows large variations, with an amplitude up to a factor 4 on time scales of a few tens of ks. We exposure corrected the EPIC-pn light curve, with the task epiclccorr and calculated the fractional variability in the $0.2-0.5,0.5-2$ and $2-10 \mathrm{keV}$ net (background subtracted) light curves with $e k$ stest, which uses algorithms developed in Edelson et al. (2002) and Vaughan (2003). The light curve shows 20-25\% variability, consistent within the errors, across the energy bands (Fig. 7). Despite the apparent oscillations, a PSD analysis of the light curve does not give a significant signal of periodicity.

An estimate of the black hole mass may be obtained from the characteristics of the short-term variability. We have calculated the excess variance of the variability, as defined in Ponti et al. (2012), from three uninterrupted $10 \mathrm{ks}$ segments of the 201404-30 XMM-Newton 0.2-10 keV light curve, with $60 \mathrm{~s}$ binning. Using the scaling relations of Ponti et al. (2012), we find a black hole mass of $M_{\mathrm{BH}}=3.5_{-2.4}^{+6.5} \times 10^{6} M_{\odot}$.

The minimum doubling time of the flux in this observation is 400 seconds, which by light travel time arguments constrains the size of the emitting region to a radius of 400 light seconds or $1.2 \times 10^{13} \mathrm{~cm}$. This is equivalent to $<8-73 R_{\mathrm{g}}$ for a non-spinning black hole in this mass range. 
The second observation is somewhat less variable; ekstest gives a fractional variability of $10.2 \pm 3.0 \%$ over the full $0.2-$ $10 \mathrm{keV}$ band. An excess variance analysis of a $10 \mathrm{ks}$ segment of the $0.2-10 \mathrm{keV}$ light curve of this observation, with $60 \mathrm{~s}$ binning, gives a weak constraint on the black hole mass of $M_{\mathrm{BH}}>6 \times$ $10^{5} M_{\odot}(90 \%$ confidence $)$, consistent with that obtained from the first observation.

\subsection{UV light curve}

During the two pointed XMM-Newton observations, the optical monitor (OM) cycled between the $B, U, U V W 1$ filters. SwiftUVOT observations were performed with the $u v w 1, u v m 2$ and $u v w 2$ filters, except for the first two observations which just used the $u v w 2$ filter. The galaxy was detected in all of the filters. Relative filter fluxes were determined using several nearby sources of comparable brightness as references. The absolute flux scale was taken from the Swift-UVOT filters, with the XMM-Newton-OM UVW1 points scaled to these by a factor 1.5.

In Fig. 4 we see that the flux, in all the UV filters, has responded to a strong flare coeval with the X-ray peak. After the flare the flux declined in all the filters, with the $u v w 2(2120 \AA)$ reducing by a factor $12 \pm 3$ or $2.7 \pm 0.3 \mathrm{mag}$, to $m=19.0$ in 524 days.

GALEX observed the galaxy on 2011-05-16 finding an NUV $(2267 \AA)$ magnitude of $20.14 \pm 0.15$ and flux of $4.2 \pm 0.6 \times$ $10^{-13} \mathrm{ergs} \mathrm{s}^{-1} \mathrm{~cm}^{-2}$. The source was not detected in the FUV (1516 ̊) band. The NUV filter has a similar bandpass to the $u v w 2$ filter and its flux is one magnitude lower than the last $u v w 2$ observation of Swift, suggesting that the flux was still decaying in this filter on 2015-09-25. We can use the GALEX measurement to subtract the contribution of the galaxy from the $u v w 2$ observations of the flare. The resulting $u v w 2$ light curve (shown in Fig. 5) has a shallower decay index than the canonical $t^{-5 / 3}$ and may be better modelled with an index $t^{-1.2}$ (Fig. 5).

The $u v w 2, u v m 2$ and $u v w 1$ light curves show signs of a recovery in flux around day 180 , which may be echoed in the X-ray curves. Similar secondary bumps have been noted in candidate TDE light curves before (e.g. Merloni et al. 2015).

\subsection{X-ray spectral analysis}

We extracted spectra from the first XMM-Newton pointed observation (hereafter XMM1), taken 29 days after the peak flux seen on 2014-04-01, from circles with optimum radii determined by the task eregionanalyse for the EPIC-pn and MOS-1 cameras ${ }^{1}$. Background spectra were created from nearby sourcefree regions ${ }^{2}$. The source spectra were grouped to have a minimum of 25 counts per bin and were fitted simultaneously within the XSPEC package (v12.8.2). Fits were performed using the Chi-squared statistic over the energy range $0.3-10 \mathrm{keV}$, using a constant to account for the small differences in normalisation between the instruments. The XMM1 observation had 16500 background subtracted counts in the two cameras and in this energy range, while the second pointed observation (hereafter XMM2) had 4700 background subtracted counts. Quoted errors are $90 \%$ confidence unless otherwise stated.

\footnotetext{
1 MOS-2 data were not used in this analysis as the small window mode data have lower statistics than MOS-1.

2 As the observation was split into three segments (see Sect. 3), source and background spectra and effective area files were extracted individually for each segment and combined using the ftools tasks mathpha and addarf.
}
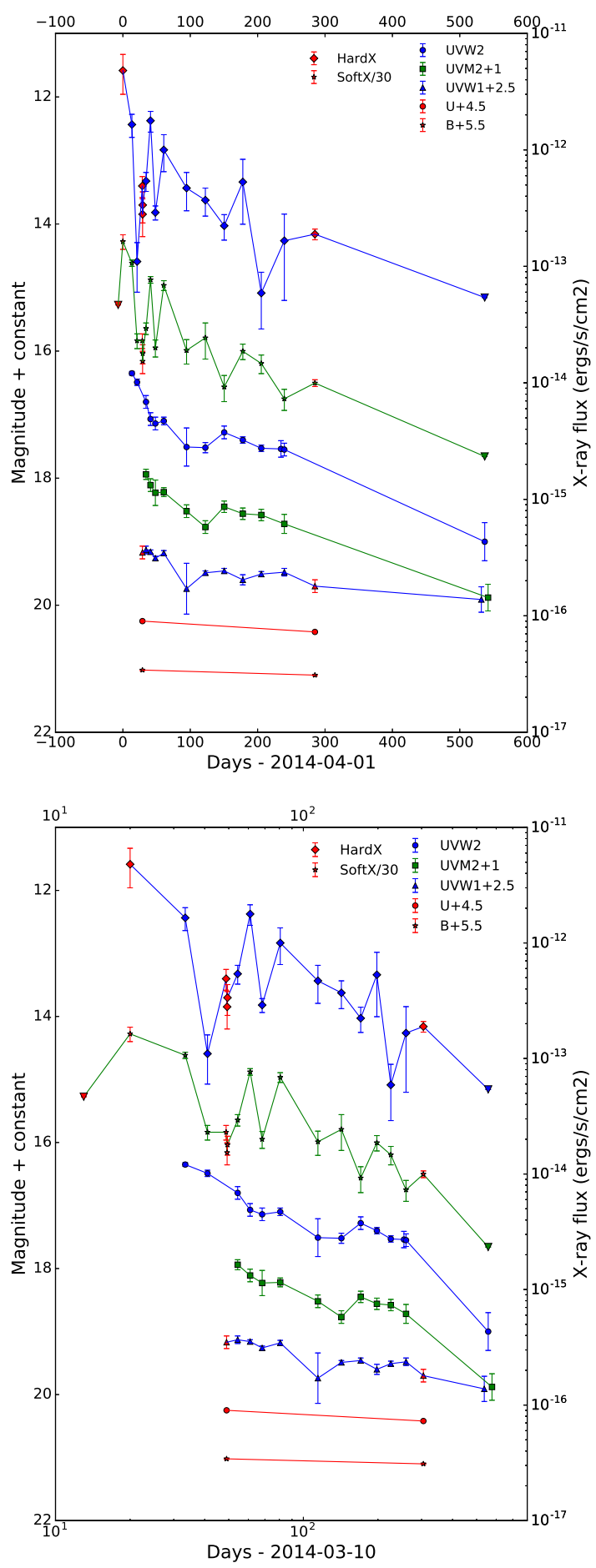

Fig. 4. Long-term X-ray and UV light curve of XMMSL1 J0740-85 plotted against linear time (upper panel) and log time (lower panel) axes. From top to bottom, $2-10 \mathrm{keV}, 0.2-2 \mathrm{keV}$, Swift-uvw2 (1928 ̊̊), Swift-uvm2 (2246 ̊), Swift-uvwl/XMM-OM-UVW1 (2600 ̊), XMMOM-U (3480 ̊), XMM-OM-B (4500 ̊). XMM-Newton points are marked in red and Swift points in blue or green. Upper limits are indicated by downwards triangles.

As a first step we fit the XMM1 observation with a simple power-law model and galactic absorption of $1.5 \times 10^{21} \mathrm{~cm}^{-2}$, modelled by TBABS with element abundances set to those in Wilms et al. (2000). The fit is poor $\left(\chi_{r}^{2}=1774 / 399\right)$ with a slope 

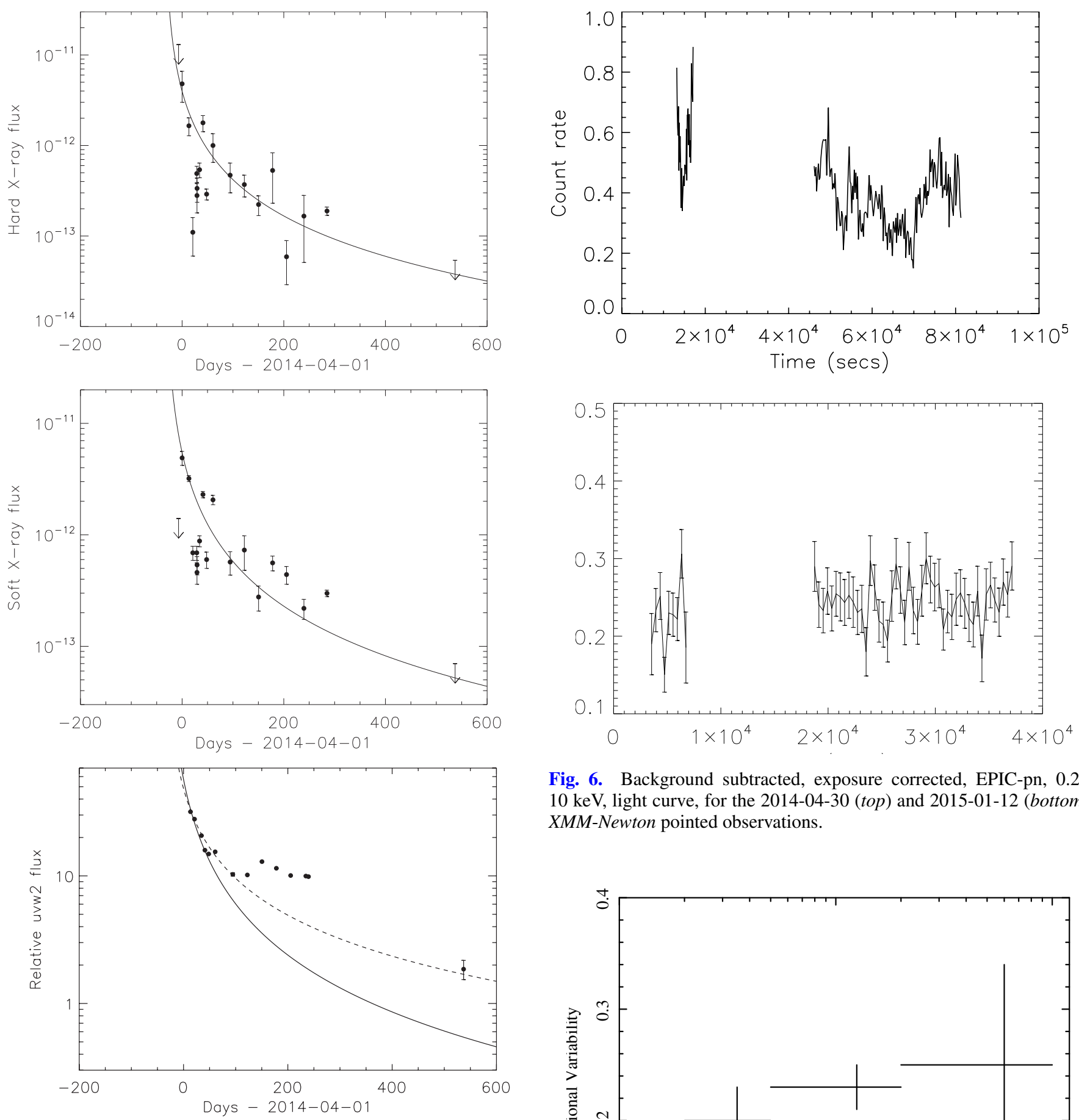

Fig. 6. Background subtracted, exposure corrected, EPIC-pn, 0.2$10 \mathrm{keV}$, light curve, for the 2014-04-30 (top) and 2015-01-12 (bottom) XMM-Newton pointed observations.

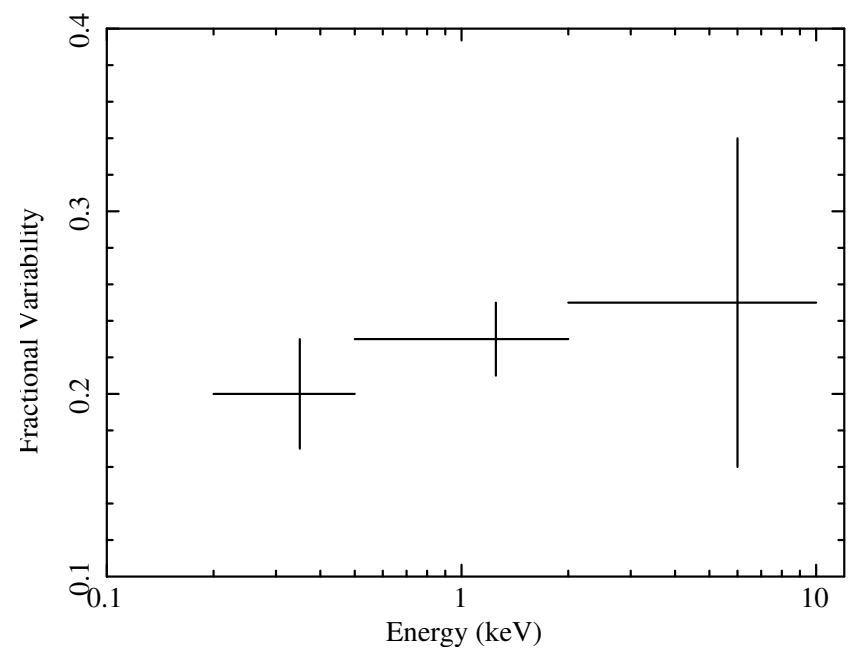

Fig. 5. Top: hard X-ray light curve overlaid with a model of $F_{2-10}=$

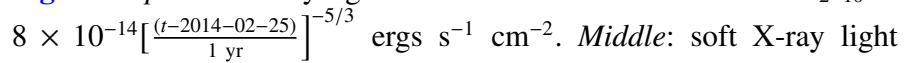
curve overlaid with a model of $F_{0.2-2}=1.1 \times 10^{-13}\left[\frac{(t-2014-02-25)}{1 \mathrm{yr}}\right]^{-5 / 3}$ ergs s $\mathrm{cm}^{-1}$. Bottom: Swift-uvw2 light curve, after subtraction of the galaxy background from a GALEX NUV observation of 2011-05-16, overlaid with a model of $F_{U V W 2} \propto\left[\frac{(t-2014-02-25)}{1 \mathrm{yr}}\right]^{-5 / 3}$ (solid curve) and a better fitting model of $F_{U V W 2} \propto\left[\frac{(t-2014-02-25)}{1 \mathrm{yr}}\right]^{-1.2}$ (dashed curve).

of $\Gamma \sim 4$. If we fit over the $2-10 \mathrm{keV}$ range only, the slope flattens to $1.76 \pm 0.11$ and large residuals are present at lower energies.

We attempted to model the low-energy excess as an extra emission component, most successfully with a blackbody component of $k T=86 \pm 2 \mathrm{eV}\left(\chi_{r}^{2}=415 / 397\right)$. Neither intrinsic

absorption $\left(\chi_{r}^{2}=460 / 396\right)$ nor relativistically-blurred reflection from an ionised disc $\left(\chi_{r}^{2}=664 / 396\right)$ gave as good a fit (see Table 2 for details). Good fits could be obtained by adding a further component. A power-law with two intrinsic absorbers $\left(\chi_{r}^{2}=393 / 393\right)$ or a power-law plus a black-body with an 
intrinsic absorber $\left(\chi_{r}^{2}=383 / 394\right)$ modelled the X-ray spectrum adequately.

A black-body temperature of $k T=86 \mathrm{eV}$ is significantly lower than the $k T \sim 120 \mathrm{eV}$ equivalent temperature ubiquitously found for the soft-excess component of AGN (e.g. Done et al. 2012) but is similar to the temperature of thermal emission which might be expected from material in the inner disc about a black hole of $10^{6} M_{\odot}$. Based on this we included the UV filters, OMUVW1 and Swift-uvm2 in the fit, with intrinsic absorption given by $B-V=0.14$ (Schlafly \& Finkbeiner 2011), to test whether they represent the low-energy part of disc emission. The Swift$u v m 2$ filter flux was taken from a linear extrapolation of the $u v m 2$ light curve to the date of the XMM1 observation (the first $u v m 2$ measurement was actually taken 5 days later). As these two UV filters show large flares, they are clearly dominated by nuclear emission, and no attempt was made to subtract any emission from the stellar population.

We changed to the optxagnf multi-coloured disc model (Done et al. 2012) which assumes that the disc emits a colourcorrected blackbody down to a given radius, within which a two-phase plasma forms a corona. The model is energetically self-consistent with accretion energy powering thermal emission from the disc, a soft X-ray excess produced by Comptonisation in optically thick plasma, and a high-energy power law produced by Comptonisation in an optically thin plasma. We first assumed a pure thermal accretion disc model, with a power-law component but no soft excess from optically-thick Comptonisation but were unable to get a good fit. We then allowed the optically thick Comptonisation to vary and found an acceptable fit with the soft X-ray excess, over a hard power-law, being provided by both thermal emission and the Comptonisation component $\left(\chi_{r}^{2}=404 / 395\right.$; Fig. 8; Table 3$)$. In this fit the black hole mass, is only very loosely constrained to $M_{\mathrm{BH}}<2 \times 10^{7} M_{\odot}(90 \%$ confidence) and so we fixed it to the best fit value from the variability analysis, $M_{\mathrm{BH}}=3.5 \times 10^{6} M_{\odot}$. A non-spinning black hole was assumed $(a=0)$. Although the fit is quite good, an excess is clearly present in the residuals between $4-6 \mathrm{keV}$. We attempted to model this with a distant reflector, using the pexmon (Nandra et al. 2007) model. This gave a small improvement of $\delta \chi^{2}=5$ for two degrees of freedom (d.o.f.), with the redshift frozen to that of the galaxy. As the residuals appear to be broad we then tried the diskline model (Laor; Laor 1991) which gave a better fit $\left(\delta \chi^{2}=22\right.$ for two d.o.f.; F-test $\left.=99.998 \%\right)$ with a line energy of $5.85 \mathrm{keV}$ in the source rest frame, which would indicate a redshift of $\sim 0.09$ if the line is due to neutral $\mathrm{Fe} \mathrm{K}_{\alpha}$. As an alternative we fit the spectrum with a partially-covering intrinsic ionised absorber (zxipcf; Reeves et al. 2008) which also gave a good result $\left(\delta \chi^{2}=19\right.$ for three d.o.f.; F-test $\left.=99.989 \%\right)$. For this model, the accretion rate is $\dot{m}=0.047_{-0.011}^{+0.009} \dot{m}_{\text {edd }}$ for a Schwarzchild black hole of $M_{\mathrm{BH}}=3.5 \times 10^{6} M_{\odot}$. Note that the accretion rate during the 2014-04-01 slew observation will have been roughly 10 times higher than this.

If we now look at the XMM2 spectra, taken 285 days after discovery, then we can see that the major change is a reduction in the observed flux below $2 \mathrm{keV}$ and a reduction, or removal, of the 4-6 keV excess seen in XMM1 (Fig. 9). We again simultaneously fit the EPIC-pn, MOS-1, OM-UVW1 and extrapolated Swift-uvm2 datasets with the optxagn model and the same fixed values of $M_{\mathrm{BH}}$ and spin. The fit is reasonable, $\chi_{r}^{2}=117 / 105$. In this case no significant improvement was found by adding an additional intrinsic absorption or broad line component (Table 3 ). From the fits we find that the accretion rate has reduced in XMM2 to $\dot{m}=0.015_{-0.006}^{+0.008} \dot{m}_{\text {edd }}$.

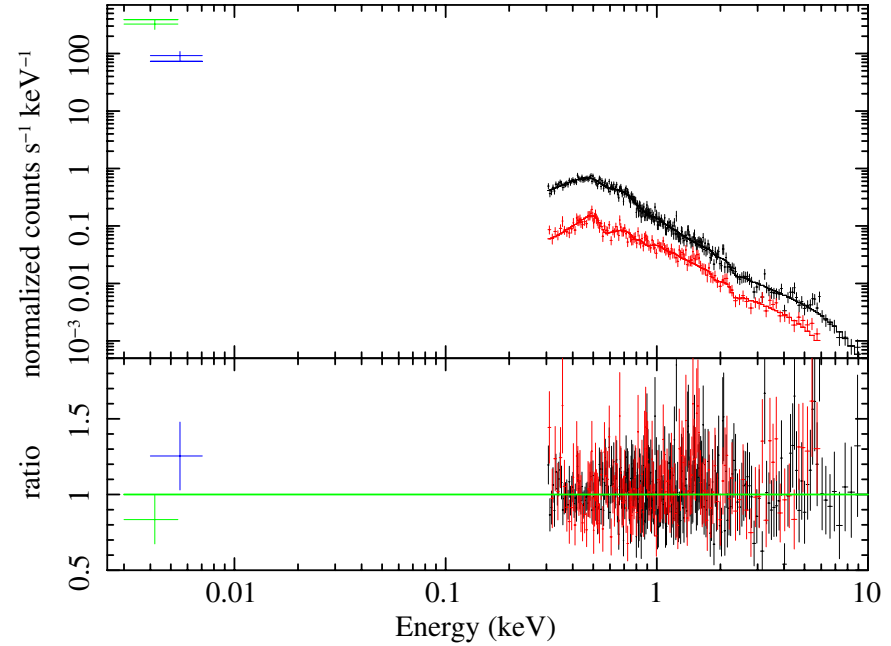

Fig. 8. A fit of a multi-colour disc model (optxagn), absorbed by the Galactic column, to the EPIC-pn (black), MOS-1 (red) and OM-UVW1 (blue) data from the XMM-Newton pointed observation of 2014-04-30 and to the Swift-uvm2 filter flux, extrapolated to the same date (green; see text).

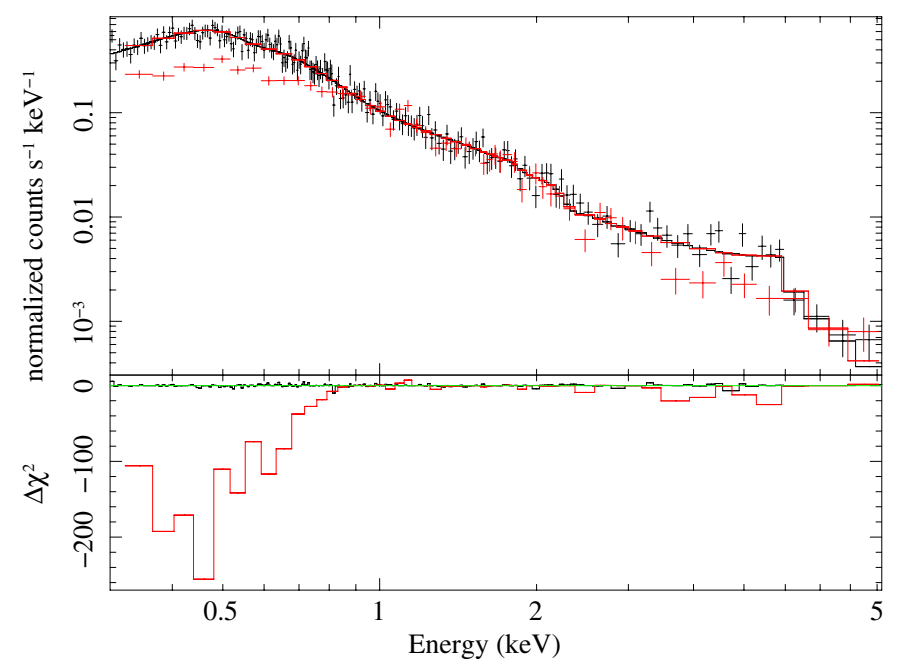

Fig. 9. A comparison of EPIC-pn spectra from the XMM1 and XMM2 pointed observations of 2014-04-30 (black) and 2015-0112 (red). The lower panel shows the residuals from the model fit, TBABS*optxagn*zxipcf to the 2014-04-30 spectrum.

\section{Discussion}

The lack of emission lines in the optical spectrum taken during the 2MASS redshift survey make it unlikely that XMMSL1 J0740-85 was a steadily-emitting AGN before the 2014 flare. We have confirmed this diagnosis with a further optical spectrum which allows us to constrain the luminosity of the [OIII] $\lambda 5007$ line to $L_{\text {OIII }}<4 \times 10^{-15} \mathrm{ergs} \mathrm{s}^{-1} \mathrm{~cm}^{-2}$. The unabsorbed 2-10 keV flux from the XMM-Newton slew observation of 2014-04-01, $F_{2-10 \mathrm{keV}} \sim 4.8 \times 10^{-12} \mathrm{ergs} \mathrm{s}{ }^{-1} \mathrm{~cm}^{-2}$, gives a ratio $L_{2-10 \mathrm{keV}} / L_{\mathrm{OIII}}>1000$, well in excess of the ratio $\sim 1-100$ found in samples of Seyfert galaxies (Panessa et al. 2006; Lamastra et al. 2009). The WISE colours, $W 1-W 2=0.0$, are also suggestive of a non-active galaxy (Stern et al. 2012).

With only a single epoch of observations, an association between the radio emission and XMMSL1 J0740-85 is necessarily tentative. There are no archival radio observations of the host of XMMSL1 J0740-85 but from the above analysis 
Table 2. Spectral fits to XMM-Newton pointed observations of XMMSL1 J0740-85.

\begin{tabular}{|c|c|c|c|c|c|c|c|c|c|c|}
\hline \multicolumn{2}{|c|}{ Power-law $^{a}$} & \multirow{2}{*}{$\begin{array}{c}\text { Power-law }^{b} \\
\Gamma\end{array}$} & \multirow{2}{*}{$\begin{array}{c}\text { Bbody }^{c} \\
k T \\
\mathrm{eV}\end{array}$} & \multirow{2}{*}{$\begin{array}{c}\text { Brem }^{d} \\
k T \\
\mathrm{eV}\end{array}$} & \multicolumn{2}{|c|}{ Reflection $^{e}$} & \multicolumn{3}{|c|}{ zxipcf $f^{f}$} & \multirow[t]{2}{*}{ C/d.o.f. ${ }^{g}$} \\
\hline$\Gamma$ & $\begin{array}{c}\text { Norm } \\
\mathrm{keV}^{-1} \mathrm{~cm}^{-2} \mathrm{~s}^{-1}\end{array}$ & & & & $\begin{array}{l}\text { Norm } \\
\times 10^{-8}\end{array}$ & $\begin{array}{c}x i \\
\operatorname{erg~cm} / \mathrm{s}\end{array}$ & $\begin{array}{c}N_{\mathrm{H}} \\
10^{22} \mathrm{~cm}^{-2}\end{array}$ & $\begin{array}{c}x i \\
\log \end{array}$ & $\begin{array}{l}\mathrm{cf} \\
\%\end{array}$ & \\
\hline \multicolumn{11}{|c|}{ XMM-Newton observation 1 - 2014-04-30 (XMM1) } \\
\hline $4.61 \pm 0.08$ & $1.26 \pm+0.08 \times 10^{-4}$ & $1.43 \pm 0.09$ & \multirow{6}{*}{$86 \pm 2$} & \multirow{6}{*}{$170 \pm 5$} & \multirow{6}{*}{\multicolumn{2}{|c|}{$2.4_{-0.4}^{+1.4} \quad 855_{-260}^{+150}$}} & & & & $516 / 397$ \\
\hline $1.94 \pm 0.06$ & $1.13 \pm 0.05 \times 10^{-4}$ & & & & & & & & & $415 / 397$ \\
\hline $1.82 \pm 0.06$ & $0.98 \pm 0.06 \times 10^{-4}$ & & & & & & & & & $416 / 397$ \\
\hline $1.48 \pm 0.06$ & $5.8 \pm 0.4 \times 10^{-5}$ & & & & & & & & & $664 / 396$ \\
\hline $3.31 \pm 0.04$ & $1.72 \pm 0.21 \times 10^{-3}$ & & & & & & $17.5_{-1.0}^{+0.9}$ & $1.95 \pm 0.02$ & $92 \pm 1$ & $460 / 396$ \\
\hline $2.45 \pm 0.20$ & $4.0_{-1.3}^{+1.9} \times 10^{-4}$ & & & & & & $18.0_{-2.9}^{+4.2}$ & $1.96_{-0.38}^{+0.08}$ & $68_{-13}^{+10}$ & $383 / 394$ \\
\hline \multicolumn{11}{|c|}{ XMM-Newton observation $2-2015-01-12$ (XMM2) } \\
\hline $4.15_{-0.33}^{+0.42}$ & $6.7_{-2.1}^{+2.8} \times 10^{-4}$ & $1.97 \pm 0.21$ & \multirow{5}{*}{$85 \pm 7$} & \multirow{5}{*}{$177 \pm 24$} & & & & & & $115 / 105$ \\
\hline $2.38 \pm 0.11$ & $1.10_{-0.09}^{+0.11} \times 10^{-4}$ & & & & & & & & & $122 / 105$ \\
\hline $2.28 \pm 0.13$ & $1.04_{-0.12}^{+0.12} \times 10^{-4}$ & & & & & & & & & $116 / 105$ \\
\hline $3.07 \pm 0.06$ & $5.2_{-1.5}^{+2.1} \times 10^{-4}$ & & & & & & $14_{-9}^{+4}$ & $1.8_{-1.4}^{+0.8}$ & $74_{-11}^{+7}$ & $122 / 104$ \\
\hline $2.79 \pm 0.24$ & $2.84_{-1.04}^{+1.07} \times 10^{-4}$ & & & & & & $6_{-4}^{+108}$ & $-0.55_{-0}^{+2.38}$ & $61_{-38}^{+18}$ & $116 / 102$ \\
\hline
\end{tabular}

Notes. All fits include absorption by the Galactic column (model TBABS, $N_{\mathrm{H}}=1.5 \times 10^{21} \mathrm{~cm}^{-2}$, abundances from Wilms et al. 2000). Errors are $90 \%$ confidence. ${ }^{(a)}$ Continuum power-law with photon index and normalisation, ${ }^{(b)}$ slope of secondary power-law modelling high-energy emission, ${ }^{(c)}$ black-body model temperature, ${ }^{(d)}$ bremsstrahlung model temperature, ${ }^{(e)}$ ionized reflection (reflionx; Ross \& Fabian 2005) with ionization state and normalisation, convolved with a relativistic blurring model (kdblur; Laor 1991; Fabian et al. 2002), with fixed emissivity index $=3$, inner radius $=4.5 G M / c^{2}$, outer radius $=100 \mathrm{GM} / \mathrm{c}^{2}$ and inclination $=45^{\circ},{ }^{(f)}$ ionized absorption (zxipcf; Reeves et al. 2008) with equivalent column density, ionization state and covering fraction, ${ }^{(g)} C$-statistic/number of degrees of freedom.

Table 3. Fits with a multi-coloured disc model to XMM-Newton pointed observations of XMMSL1 J0740-85.

\begin{tabular}{|c|c|c|c|c|c|c|c|c|c|c|}
\hline \multicolumn{5}{|c|}{ optxagnf $^{a}$} & \multicolumn{2}{|c|}{ Disc line $^{b}$} & \multicolumn{3}{|c|}{ zxipcf ${ }^{c}$} & \multirow[t]{2}{*}{$C /$ d.o.f. } \\
\hline $\begin{array}{c}\dot{m} \\
\log \left(\dot{m}_{\mathrm{edd}}\right)\end{array}$ & slope & $\begin{array}{c}k T \\
(\mathrm{eV})\end{array}$ & $\tau_{\mathrm{e}}$ & $f_{\mathrm{pl}}$ & $\begin{array}{c}E_{\text {line }} \\
(\mathrm{keV})\end{array}$ & $\begin{array}{l}\text { Norm } \\
\times 10^{-6} \\
\end{array}$ & $\begin{array}{c}N_{\mathrm{H}} \\
10^{22} \mathrm{~cm}^{-2}\end{array}$ & $\begin{array}{c}x i \\
\log \end{array}$ & $\begin{array}{l}\mathrm{cf} \\
\%\end{array}$ & \\
\hline \multicolumn{11}{|c|}{ XMM-Newton observation 1 - 2014-04-30 (XMM1) } \\
\hline$-1.37_{-0.16}^{+0.12}$ & $1.87 \pm 0.07$ & $101_{-8}^{+9}$ & $33_{-8}^{+19}$ & $0.25_{-0.10}^{+0.09}$ & \multirow{3}{*}{$5.76_{-0.14}^{+0.17}$} & \multirow{3}{*}{$5.36_{-1.86}^{+1.81}$} & & & & $404 / 395$ \\
\hline$-1.36_{-0.17}^{+0.12}$ & $2.06 \pm 0.10$ & $92_{-5}^{+9}$ & $49_{-20}^{+51}$ & $0.28_{-0.03}^{+0.09}$ & & & & & & $382 / 393$ \\
\hline$-1.33_{-0.11}^{+0.08}$ & $1.84 \pm 0.09$ & $153_{-28}^{+27}$ & $17_{-3}^{+5}$ & $0.13_{-0.07}^{+0.05}$ & & & $0.7_{-0.3}^{+1.8}$ & $2.16_{-0.33}^{+0.21}$ & $>63$ & $383 / 392$ \\
\hline \multicolumn{11}{|c|}{ XMM-Newton observation 2 - 2015-01-12 (XMM2) } \\
\hline$-1.81_{-0.24}^{+0.18}$ & $2.30 \pm 0.12$ & $114 \pm 20$ & $24_{-6}^{+19}$ & $0.24_{-0.12}^{+0.24}$ & \multirow{3}{*}{$6.84_{-0.3}^{+0.4}$} & \multirow{3}{*}{$1.7_{-1.7}^{+2.0}$} & & & & $117 / 105$ \\
\hline$-1.87_{-0.24}^{+0.16}$ & $2.37 \pm 0.13$ & $106_{-4}^{+6}$ & $25_{-7}^{+52}$ & $0.28_{-0.13}^{+0.42}$ & & & & & & $116 / 103$ \\
\hline$-1.83_{-0.2}^{+0.2}$ & $2.32_{-0.17}^{+0.20}$ & $110_{-15}^{+35}$ & $25_{-8}^{+25}$ & $0.27_{-0.19}^{+0.28}$ & & & $15_{-8}^{+10}$ & $<6$ & $<1$ & $117 / 102$ \\
\hline
\end{tabular}

Notes. All fits include absorption by the Galactic column (model TBABS, $N_{\mathrm{H}}=1.5 \times 10^{21} \mathrm{~cm}^{-2}$ ). Errors are $90 \%$ confidence. ${ }^{(a)}$ Shown are parameters of the optxagnf model (Done et al. 2012) with black hole mass fixed to $M_{\mathrm{BH}}=3.5 \times 10^{6} M_{\odot}$ and spin to $a=0$; accretion rate, power-law slope, electron temperature of the soft Comptonisation component, optical depth, the disc radius, within which emission changes from black-body to a Comptonised spectrum, and the fraction of energy emitted as a power-law; ${ }^{(b)}$ observed energy of a relativistically broadened line given by the (Laor model; Laor 1991); ${ }^{(c)}$ ionized absorption with equivalent column density, ionization state and covering fraction $(z x i p c f)$.

it is unlikely that the radio emission is due to AGN activity. The stellar population synthesis modeling discussed in Sect. 4 suggests that the radio emission is also unlikely to be due to ongoing star formation. The upper limit on OIII emission of $4 \times 10^{-15} \mathrm{ergs} \mathrm{s}^{-1} \mathrm{~cm}^{-2}$ corresponds to a star formation rate of $<0.4_{-0.3}^{+0.6} M_{\odot} /$ year (Moustakas et al. 2006). This rate implies a radio flux density of $<0.5_{-0.3}^{+0.7} \mathrm{mJy}$ at $1.5 \mathrm{GHz}$ (Condon et al. 2002). A more restrictive estimate of the current star formation rate can be obtained from the 2011 NUV detection of the host galaxy by GALEX (Sect. 6.1). Following the method of Wilkins et al. (2012), we estimate the star formation rate to be $\sim 0.02 M_{\odot} /$ year. This rate implies a $1.5 \mathrm{GHz}$ radio flux density of $\sim 0.03 \pm 0.01 \mathrm{mJy}$, which is a factor of 40 less than the flux we observe.

Scaling the best fitting spectral model from the XMM1 observation to the 2014-04-01 XMM-Newton slew count rate gives 


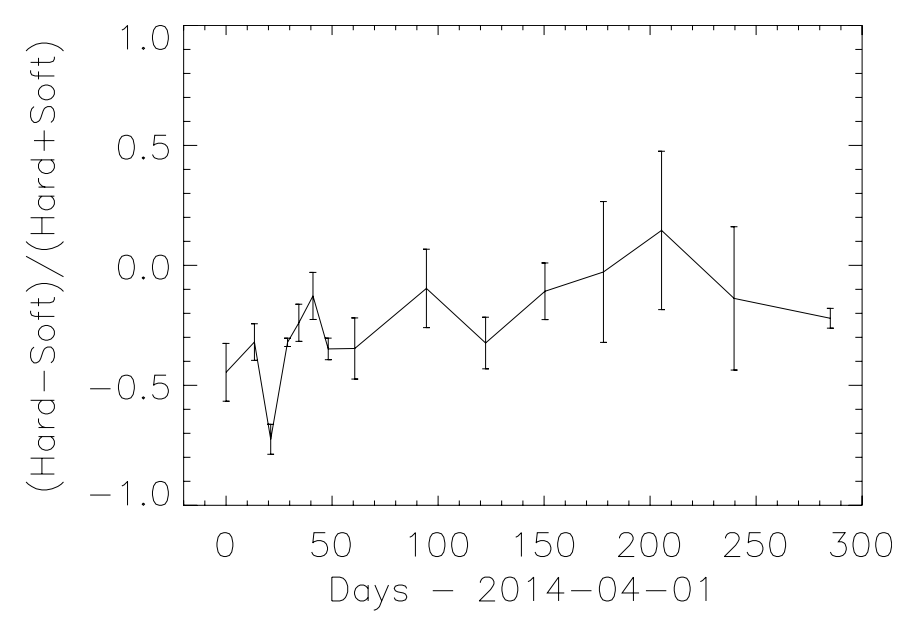

Fig. 10. $2-10 \mathrm{keV}$ to $0.2-2.0 \mathrm{keV}$ flux ratio, calculated by fitting a phenomenological model of a power-law plus black-body absorbed by the Galactic column.

$L_{\text {bol }} \sim 2 \times 10^{44} \mathrm{ergs} \mathrm{s}^{-1}$. As this is in excess of even the brightest ULX, the conclusion is that the SMBH of XMMSL1 J074085 has experienced a sudden increase in available fuel, probably from the tidal disruption of a stellar object.

From the relationship of black hole mass to bulge $K$-band luminosity (Marconi \& Hunt 2003) we find $M_{\mathrm{BH}} \sim 6 \times 10^{6} M_{\odot}$ using the point source $2 \mathrm{MASS}$ magnitude of $m_{K}=11.72$, with a systematic error of $0.3 \mathrm{dex}\left(2 \times 10^{6} M_{\odot}\right)$. This is consistent with the value of $M_{\mathrm{BH}}=3.5_{-2.4}^{+6.5} \times 10^{6} M_{\odot}$ derived from the X-ray variability study (Sect. 6).

The total energy release, integrating between the discovery date and the XMM 2 observation 285 days later is $\sim 5 \times 10^{50} \mathrm{ergs}$, equivalent to a consumed mass of $0.025 M_{\odot}$ assuming a conversion efficiency of 0.1 .

The fact that we cannot fit the UV and soft X-ray excess with a single thermal disc model implies that they are not part of the same thermal component. This is in common with many TDE where the UV emission appears to be the high-energy part of a separate, lower-temperature, optical component (Gezari et al. 2012; Holoien et al. 2016a,b).

In the late stages of a flare the X-ray spectrum has been seen to harden on several occasions (Vaughan et al. 2004; Komossa \& Bade 1999a; Komossa et al. 2004). At peak, however, most TDE discovered in the soft X-ray band did not have significant emission above $\sim 2 \mathrm{keV}$ (e.g. Komossa \& Bade 1999a; Esquej et al. 2008; Saxton et al. 2012; Lin et al. 2015). XMMSL1 J0740-85 has comparable flux in the soft and hard X-ray bands (Fig. 10) and is a clear example, without significant internal absorption, of a TDE with strong thermal and power-law emission.

It is not yet completely demonstrated whether powerlaw X-ray emission in TDE is produced in a jet or an AGN-like comptonisation region. While the luminosity of XMMSL1 J0740-85 is below that of the proposed Swift on-axis relativistic jets it is comparable with NGC 4845, whose hard ( $\Gamma \sim 2.2$; Nikolajuk \& Walter 2013) X-ray emission has been ascribed to an off-axis jet (Lei et al. 2016) based on its radio emission (Irwin et al. 2015). The detection of radio emission from XMMSL1 J0740-85 may point in this direction. However, the detection of spectral features around $6 \mathrm{keV}$, whether due to intrinsic absorption or ionised reflection, are more suggestive of non-jetted intrinsic emission. In this context the radio emission could be interpreted as a non-relativistic outflow akin to that seen in ASSASN-14li (Alexander et al. 2016). The nature of the radio emission in XMMSL1 J0740-85 will be explored further in Alexander et al. (in prep.).

It has recently been discovered that a large fraction of optically-selected TDE occur in host galaxies which have experienced a starburst that has recently finished (Arcavi et al. 2014; French et al. 2016). From detailed stellar population modelling in Sect. 4 we showed that the host galaxy of XMMSL1 J074085 has undergone a period of strong star formation about $2 \mathrm{Gyr}$ ago. French et al. (2016) use line diagnostics to identify these galaxies by the presence of an $\mathrm{H}_{\delta}$ absorption line with equivalent width $(E W)>3 \AA$ and an $\mathrm{H}_{\alpha}$ emission line with $E W<3 \AA$. We measure an EW, in absorption, of $\mathrm{H}_{\delta} \sim 3 \AA$ from the CTIO2MASS (pre-flare) spectrum and determine a strict upper limit to the $H_{\alpha}$ emission of $<1 \AA$, from the, higher statistic, Las Campanas spectrum after correcting for stellar absorption. Therefore, this TDE also appears to be hosted in a rare post-starburst galaxy.

\section{Summary}

A flare was detected from the galaxy XMMSL1 J0740-85 on April 1st 2014, reaching a bolometric luminosity, $L_{\mathrm{bol}}=2 \times$ $10^{44} \mathrm{ergs} \mathrm{s}^{-1}$. The source flux subsequently decayed by a factor $>70$ in the 2-10 and $0.2-2 \mathrm{keV} X$-ray bands and by up to $2.7 \mathrm{mag}$ in UV filters. The flux change in the optical, $B$ filter was small. While the UV emission may be thermal, we model the soft X-ray flux as an excess on top of a significant power-law component which extends out to at least $10 \mathrm{keV}$. Weak radio emission, peaking at $1.5 \mathrm{GHz}$, was detected 21 months after discovery. From the rapid X-ray variability we calculate the mass of the black hole to be $1 \times 10^{6}<M_{\mathrm{BH}}<\sim 1 \times 10^{7} M_{\odot}$ and constrain the location of the X-ray emission to within $<73 R_{\mathrm{g}}$ of the black hole. The galaxy shows no signs of previous AGN activity and we attribute the flare to the accretion of debris from a star which was tidally destroyed during a close approach to the nuclear black hole. XMMSL1 J0740-85 is perhaps the cleanest example yet found of a TDE emitting both thermal and non-thermal radiation. It continues the recently-discovered trend of TDE being preferentially hosted in post-starburst galaxies. If XMMSL1 J0740-85 is a TDE then its flux should continue to reduce over the coming years.

Acknowledgements. We thank the anonymous referee for useful comments which improved the paper, and the XMM OTAC for approving this program. The XMM-Newton project is an ESA science mission with instruments and contributions directly funded by ESA member states and the USA (NASA). The $X M M-N e w t o n$ project is supported by the Bundesministerium für Wirtschaft und Technologie/Deutches Zentrum für Luft- und Raumfahrt i (BMWI/DLR, FKZ 50 OX 0001), the Max-Planck Society and the Heidenhain-Stiftung. We thank the Swift team for approving and performing the monitoring observations. This work made use of data supplied by the UK Swift Science Data Centre at the University of Leicester. We are very grateful to Lucas Macri for providing the optical spectrum and calibration data taken during the 2MASS redshift survey. We thank Pilar Esquej for calculating the ROSAT upper limit, Edo Berger and Giovanni Miniutti for useful discussions and Phil Edwards for rapidly scheduling our ATCA observations. The Australia Telescope Compact Array is part of the Australia Telescope National Facility which is funded by the Australian Government for operation as a National Facility managed by CSIRO.

\section{References}

Alexander, K., Berger, E., Guillochon, J., Zauderer, B., \& Williams, P. 2016 , ApJ, 819, 25

Arcavi, I., Gal-Yam, A., Sullivan, M., et al. 2014, ApJ, 793,38 
Bade, N., Komossa, S., \& Dahlem, M. 1996, A\&A, 309, L35

Bonning, E., Cheng, L., Shields, G., Salviander, S., \& Gerbhardt, K. 2007, ApJ, 659,211

Breeveld, A., Curran, P., Hoversten, E., et al. 2010, MNRAS, 406, 1687

Bruzual, G., \& Charlot, S. 2003, MNRAS, 344, 1000

Brown, G., Levan, A., Stanway, E., et al. 2015, MNRAS, 452, 4297

Burrows, D., Hill, J., Nousek, J., et al. 2005, Space Sci. Rev., 120, 165

Cardelli, J., Clayton, G., \& Mathis, J. 1989, ApJ, 345, 245

Cenko, S., Krimm, H., Horesh, A., et al. 2012, ApJ, 753, 77

Cenko, S., Cucchiara, A., Roth, N., et al. 2016, ApJ, 818, 32

Cid Fernandes, R., Mateus, M., Sodre Jr., L., Stasinka, G., \& Gomes, J. 2005 MNRAS, 358, 363

Cid Fernandes, R., Stasinka, G., Mateus, A., \& Vale Asari, N. 2011, MNRAS, 413,1687

Cole, S., Norberg, P., Baugh, C., et al. 2001, MNRAS, 326, 255

Condon, J., Cotton, W., \& Broderick, J. 2002, AJ, 123, 188

Done, C., Davis, S., Jin, C., Blaes, O., \& Ward, M. 2012, MNRAS, 420, 1848

Donley, J., Brandt, W., Eracleous, M., \& Boller, Th. 2002, AJ, 124, 1308

Edelson, R., Turner, T., Pounds, K., et al. 2002, ApJ, 568, 610

Esquej, P., Saxton, R., Freyberg, M., et al. 2007, A\&A, 462, L49

Esquej, P., Saxton, R., Komossa, S., Read, A., \& Freyberg, M. J. 2008, A\&A, 489,543

Evans, P., Beardmore, A., Page, K., et al. 2009, MNRAS, 397, 1177

Fabian, A., Ballantyne D., Merloni, A., et al. 2002, MNRAS, 331, L35

Filippenko, A. 1982, PASP, 94, 715

French, K., Arcavi, I., \& Zabludoff, A. 2016, ApJ, 818, 21

Gabriel, C., et al. in ADASS Xiii eds. Oschenbein, F., Allen, M., \& Egret, D. ASP Conf. Ser., 314, 759 (2003).

Gehrels, N., Chincarini, G., Giommi, P., et al. 2004, ApJ, 611, 1005

Gezari, S., Martin, D., Milliard, B., et al. 2006, ApJ, 653L, 25

Gezari, S., Basa, S., Martin, D., et al. 2008, ApJ, 676, 944

Gezari, S., Heckman, T., Cenko, S., et al. 2009, ApJ, 698, 1367

Gezari, S., Chornock, R., Rest, A., et al. 2012, Nature, 485, 217

Goad, M., Tyler, L., Beardmore, A., et al. 2007, A\&A, 476, 1401

Guillochon J., Manukian H., \& Ramirez-Ruiz E. 2014, ApJ, 783, 23

Halpern, J., Gezari, S., \& Komossa, S. 2004, ApJ, 604, 572

Haardt, F., \& Maraschi, L. 1991, ApJ, 380, L51

Hills, J., 1975, Nature, 254, 295

Holoien, T., Kochanek, C., Prieto, J., 2016a, MNRAS, 455, 2918

Holoien, T., Kochanek, C., Prieto, J., et al. 2016b, MNRAS, 464, 3813

Huchra, J., Macri, L., Masters, K., et al. 2012, ApJS, 199, 26

Irwin, J., Henriksen, R., Krause, M., et al. 2015, ApJ, 809, 172

Jansen, F., Lumb, D., Altieri, B., et al. 2001, A\&A, 365, L1

Kara, E., Miller, J., Reynolds, C., \& Dai, L. 2016, Nature, 535, 7612

Komossa, S. 2002, Rev. Mod. Astron., 15, 27

Komossa, S., \& Bade, N. 1999a, A\&A, 343, 775

Komossa, S., \& Greiner, J. 1999b, A\&A, 349, L45
Komossa, S., Halpern, J., Schartel, N., et al. 2004, ApJ, 603, L17

Komossa, S., Zhou, H., Wang, T., et al. 2008, ApJ, 678, 13

Lamastra, A., Bianchi, S., Matt, G., et al. 2009, A\&A, 504, 73

Laor, A. 1991, ApJ, 376, 90

Lei, W.-H., Yuan, Q., Zhang, B., \& Wang, D. 2016, ApJ, 816, 20

Levan, A., Tanvir, N., Cenko, S., et al. 2011, Science, 333, 6039

Lin, D., Maksym, W., Irwin, J., et al. 2015, ApJ, 811, 43

Maksym, W., Ulmer, M., \& Eracleous, M. 2010, ApJ, 722, 1035

Marconi, A., \& Hunt, L. 2003, ApJ, 589, L21

Merloni, A., Dwelly, T., Salvato, M., et al. 2015, MNRAS, 452, 69

Miller, J., Kaastra, J., Miller, M., Reynolds, M., Brown, G., et al. 2015, Nature, 526,542

Moustakas, J., Kennicutt, R., \& Tremonti, C. 2006, ApJ, 642, 775

Nandra, K., O'Neill, P., George, I., \& Reeves, J. 2007, MNRAS, 382, 194

Nikolajuk, M., \& Walter, R. 2013, A\&A, 552, A75

Panessa, F., Bassani, L., Cappi, M., et al. 2006, A\&A, 455, 173

Phinney, E. S. 1989, IAU Symp., 136, 543

Ponti, G., Papadakis, I., Bianchi, S., et al. 2012, A\&A, 542, A83

Poole, T., Breeveld, A., Page, M., et al. 2008, MNRAS, 383, 627

Read, A., Saxton, R., Torres, M., et al. 2008, A\&A, 482, L1

Rees, M. J. 1988, Nature, 333, 523

Reeves, J., Done, C., Pounds, K., et al. 2008, MNRAS, 385, 108

Roming, P. W. A., Kennedy, T. E., Mason, R. O. et al. 2005, Space Sci. Rev., 120,95

Ross, R., \& Fabian, A. 2005, MNRAS, 358, 211

Saxton, R., Read, A., Esquej, P., et al. 2008, A\&A 480, 611

Saxton, R., Read, A., Esquej, P., et al. 2012, A\&A, 541, A106

Saxton, R., Read, A., Komossa, S., et al. 2014, A\&A, 572, A1

Schlafly, E., \& Finkbeiner, D. 2011, ApJ, 737, 103

Stern, D., Assef, R., Benford, D., et al. 2012, ApJ, 753, 30

Strubbe, L. E., \& Murray, N. 2015, MNRAS, 454, 2321

Strubbe, L., \& Quataert, E. 2009, MNRAS, 400, 2070

Strubbe, L. E., \& Quataert, E. 2011, MNRAS, 415, 168

Sunyaev, R., \& Titarchuk, T. 1980, A\&A, 86, 121

van Velzen, S., \& Farrar, G. 2014, ApJ, 792, 53

van Velzen, S., Farrar, G., Gezari, S. et al. 2011, ApJ, 741, 73

van Velzen, S., Anderson, G., Stone, N., et al. 2016, Science, 351, 62

Vaughan, S., Edelson, R., Warwick, R., \& Uttley, P. 2003, MNRAS, 345, 1271

Vaughan, S., Edelson, R., \& Warwick, R. 2004, MNRAS, 349, L1

Wang, T.-G., Zhou, H.-Y., Wang, L.-F., Lu, H.-L., \& Xu, D. 2011, ApJ, 740, 85

Wilkins, S., Gonzalez-Perez, V., Lacey, C., \& Baugh, C. 2012, MNRAS, 424, 1522

Willingale, R., Starling, R., Beardmore, A., Tanvir, N., \& O’Brien, P. 2013, MNRAS, 431, 394

Wilms, J., Allen, A., \& McCray, R. 2000, ApJ, 542, 914

Zauderer, B., Berger, E., Soderberg, A. et al. 2011, Nature, 476, 425 